\title{
Safety operation of chromatography column system with discharging hydrogen radiolytically generated
}

\author{
Sou Watanabe*, Yuichi Sano, Kazunori Nomura, Yoshikazu Koma, and Yoshihiro Okamoto \\ Japan Atomic Energy Agency, 4-33, Muramatsu, Tokai-mura, Naka-gun, Ibaraki 319-1194, Japan
}

Received: 30 April 2015 / Received in final form: 18 September 2015 / Accepted: 5 October 2015

Published online: 09 December 2015

\begin{abstract}
In the extraction chromatography system, accumulation of hydrogen gas in the chromatography column is suspected to lead to fire or explosion. In order to prevent the hazardous accidents, it is necessary to evaluate behaviors of gas radiolytically generated inside the column. In this study, behaviors of gas inside the extraction chromatography column were investigated through experiments and Computation Fluid Dynamics (CFD) simulation. $\mathrm{N}_{2}$ gas once accumulated as bubbles in the packed bed was hardly discharged by the flow of mobile phase. However, the CFD simulation and X-ray imaging on $\gamma$-ray irradiated column revealed that during operation the hydrogen gas generated in the column was dissolved into the mobile phase without accumulation and discharged.
\end{abstract}

\section{Introduction}

The extraction chromatography technology is one of the promising methods for the partitioning of minor actinide (MA: Am and Cm) from spent nuclear fuel [1], and Japan Atomic Energy Agency (JAEA) has been conducting research and development for the implementation. In those studies, we carried out design of an appropriate flow sheet [2], laboratory scale separation experiments on a genuine high level liquid waste [3], development of the engineering scale apparatus [4] and inactive repeated separation experiments using the large scale apparatus [5]. In order to progress the implementation, not only the performance of the column but also the safety of this system have to be guaranteed.

In respect of the safety, fire and explosion are one of the influential accidents which should be evaluated for nuclear chemical processing including the chromatography system. They are suspected to be caused by accumulation of hydrogen gas produced by radiolysis of adsorbents or mobile phase. Since radioactive nuclides in the aqueous solution are processed by adsorbents involving organic compounds, generation of hydrogen gas caused by radiolysis of water and the organic compounds is an unavoidable phenomenon. Consequently, the generated hydrogen gas has to be safely discharged from the column for the purpose of preventing fire or explosion.

\footnotetext{
*e-mail: watanabe.sou@jaea.go.jp
}

Gas and heat are considered to be generated at the adsorption band of MA simultaneously. An increase in temperature of the mobile phase will lead to a decrease in the solubility of $\mathrm{H}_{2}$ gas into it, thus heat from radioactive elements has also to be discharged as fast as possible. Our previous study has shown that flow of the mobile phase transports the decay heat to the outside of the column [4].

In this study, generation, accumulation and discharge behavior of hydrogen gas were investigated through experiments and Computation Fluid Dynamics (CFD) simulation.

\section{Experimental}

\subsection{Behavior of gas in the engineering scale column}

The large scale testing system consists of a column, tanks and pumps as shown in Figure 1. The column of ID $200 \mathrm{~mm} \Phi$ with $650 \mathrm{~mm}$ height was used for the experiments. The column has 18 ports for sensors for measuring the electric conductivity of the mobile phase, and a gas inlet was installed at the bottom of the column. The $\mathrm{SiO}_{2}-\mathrm{P}$ support, which was prepared according to the article [6], was mixed with water in the slurry tank and transferred to the column by a mohno pump for packing.

$\mathrm{N}_{2}$ gas was supplied into the packed bed through the gas inlet, and then $\mathrm{N}_{2}$ gas discharged from the column was collected at downstream of the column as shown in Figure 2. In this measurement, amount of the supplied gas and flow 


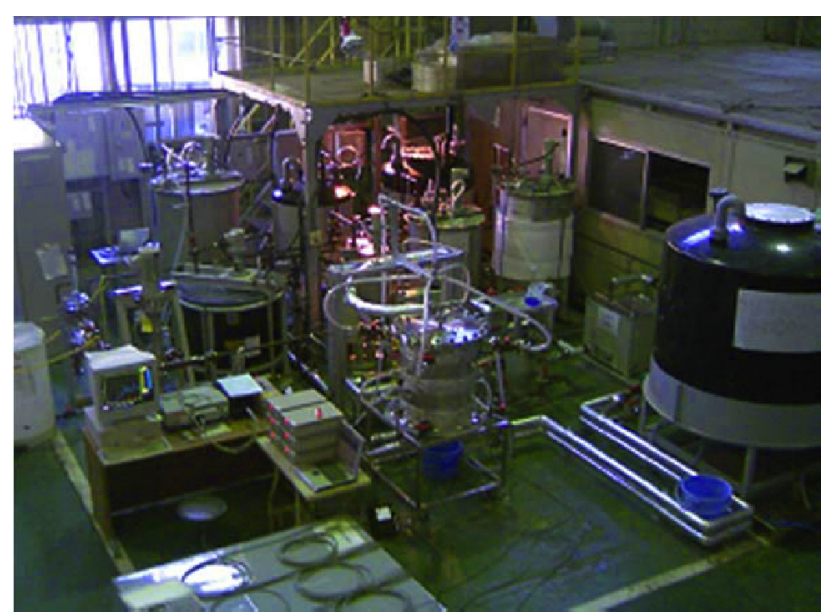

Fig. 1. Overview of the large scale system.

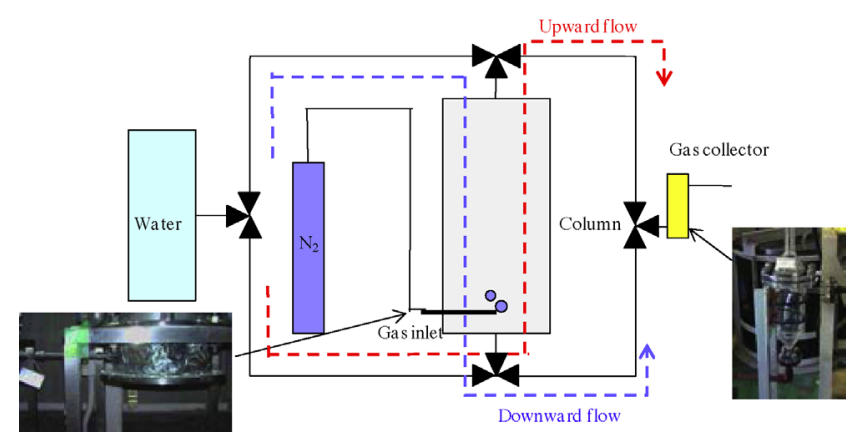

Fig. 2. Outline of the experiments for gas recovery.

direction were parametrically changed as shown in Table 1. The average flow velocity in the column was determined by detecting the change in the electric conductivity of the mobile phase when certain amount of $\mathrm{Cu}\left(\mathrm{NO}_{3}\right)_{2}$ solution was mixed in the water carrier as a tracer [4]. The tracer profiles were analyzed by the same manner with deriving the height equivalent of the theoretical plate (HETP) according to the following equations:

$$
N=2 \pi\left(\frac{t \times h}{A}\right)^{2}, H=\frac{L}{N},
$$

where $N$ is the number of the theoretical plate, $t$ is the retention time, $h$ is the height of the profile, $A$ is the area of the profile, $H$ is the HETP, $L$ is the length of the column.

Table 1. The experimental conditions for gas recovery.

\begin{tabular}{lrl}
\hline No. & Amount of $\mathrm{N}_{2}$ gas $(\mathrm{mL})$ & Flow direction \\
\hline (a) & 200 & Downward \\
(b) & 50 & Downward \\
(c) & 200 & Upward \\
(d) & 50 & Upward \\
\hline
\end{tabular}

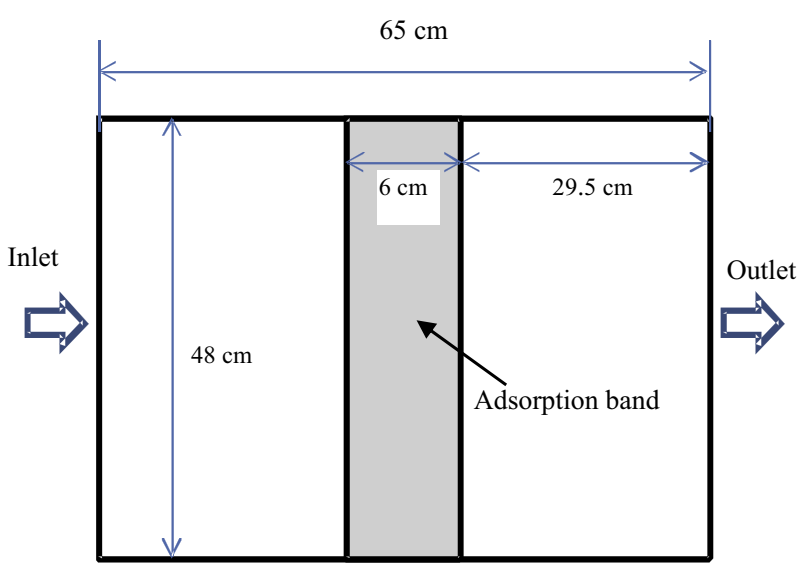

Fig. 3. Column configuration for the CFD calculation.

\subsection{CFD simulations}

Simulation on two-dimensional side view of the column with $480 \mathrm{~mm}$ ID and $650 \mathrm{~mm}$ height was carried out to evaluate the accumulation behavior of heat and gas. Two-dimensional geometry was employed in order to evaluate influence of wall on distributions of velocity, temperature inside the column. The system consists of the bed, wall, inlet and outlet of the mobile phase as shown in Figure 3. Mobile phase was water, and outlet of the column was not pressured. Uniform and immobile adsorption band of MA was assumed at middle of the column. Heat from the adsorption band, which was calculated from the decay heat of ${ }^{241} \mathrm{Am}$ and ${ }^{244} \mathrm{Cm}$, was $0.023 \mathrm{~W} / \mathrm{cm}^{3}$ and constant. Temperature of the wall was constant at $323 \mathrm{~K}$ which is one of the typical operational conditions of the extraction chromatography process [5]. In this simulation, $\mathrm{H}_{2}, \mathrm{O}_{2}, \mathrm{NO}_{2}$ and $\mathrm{CO}_{2}$ were considered as the products at the adsorption band by radiolysis. The generation rate of the gas was given by:

$$
N_{i}=3.73 \times 10^{-4} \times P \times G_{i},
$$

where $N, P$ and $G$ are amount of the generated gas $[\mathrm{mol} / \mathrm{h}]$, heat from the adsorption band $[\mathrm{W}]$ and $G$ value [molecules/ $100 \mathrm{eV}]$ of component $i$, respectively. $G$ values shown in Table 2 except for that of $\mathrm{CO}_{2}$ are taken from an article [7], and $G$ value of $\mathrm{CO}_{2}$ was estimated from the results of $\gamma$-ray irradiation experiments on the adsorbents [8]. As shown in Figure 4, generated gas was assumed to stay at the original mesh unless it dissolves into the mobile phase. Dissolution of the gas into the mobile phase follows the Henry's law [9].

Table 2. $G$ values of the gas components.

\begin{tabular}{ll}
\hline Component & $G$ value $[$ molecules $/ 100 \mathrm{eV}]$ \\
\hline $\mathrm{H}_{2}$ & 1.6 \\
$\mathrm{O}_{2}$ & 0.20 \\
$\mathrm{NO}_{2}$ & 1.1 \\
$\mathrm{CO}_{2}$ & 3.9 \\
\hline
\end{tabular}




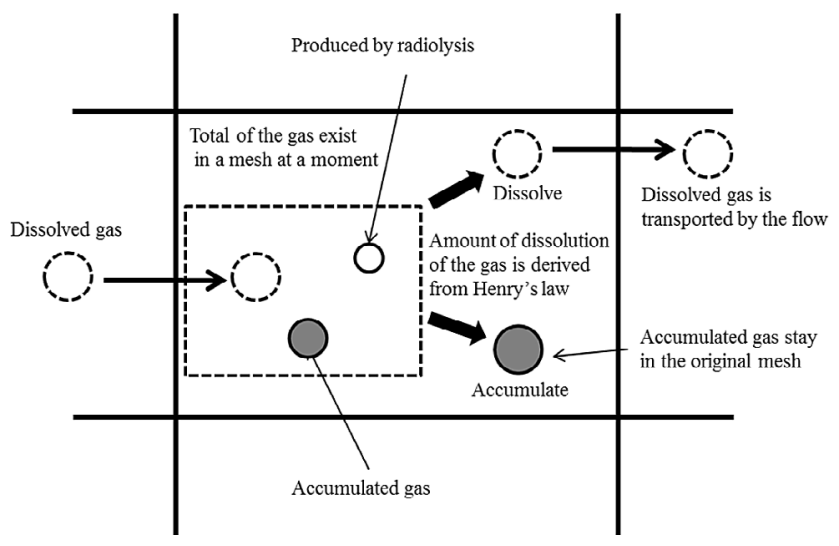

Fig. 4. Conceptual diagram of behavior of gas in the CFD calculation.

Geometry was produced by GAMBIT 2.4.6 [10] software and calculation was carried out by FLUENT 12.0 software [11]. The packed bed was simulated by water and porous media with porosity of 0.37 , and the pressure drop of the bed was proportional to the velocity of the water. The thermal conductivity and heat capacity of the bed were experimentally measured to be $\lambda_{\text {eff }}=0.525 \mathrm{~W} / \mathrm{m} \cdot \mathrm{K}$ and $C \mathrm{p}_{\text {eff }}=7.40 \mathrm{~J} / \mathrm{g} \cdot \mathrm{K}$, respectively. General features of CFD simulation are shown in Table 3. The flow velocity distribution was calculated with different mesh sizes, and an appropriate size was selected to eliminate dependence of results on the mesh.

\subsection{X-ray imaging on $\gamma$-ray irradiated columns}

The $\mathrm{CMPO} / \mathrm{SiO}_{2}-\mathrm{P}$ adsorbent contained CMPO (n-octyl (phenyl)- $N, N$-diisobutylcarbamoyl-methylphosphine oxide)

Table 3. General features of the CFD model.

\begin{tabular}{ll}
\hline Parameter & Model \\
\hline Solver & Pressure based, double precision \\
Geometry & 2-dimensional axisymmetric \\
Turbulence & Laminar flow \\
Discretization & Pressure: standardDensity: first order \\
& upwindMomentum: first order \\
& upwindTurbulent kinetic energy: first \\
& order upwindSpecific dissipation rate: \\
& No-slip \\
Walls & $323 \mathrm{~K}$ \\
Temperature & \\
of the wall & $1 \mathrm{~s}$ \\
Time step size & Uniform rectangle \\
Mesh type & $1 \mathrm{~mm} \times 1 \mathrm{~mm}$ \\
Mesh size & 156000 \\
The number & \\
of mesh & $\Delta P / L[\mathrm{kPa} / \mathrm{m}]=a \times v[\mathrm{~m} / \mathrm{s}]$, \\
Pressure drop & $a=1.45 \times 10^{5}\left[\mathrm{kPa} \cdot \mathrm{s} / \mathrm{m}^{2}\right]$ \\
of the bed &
\end{tabular}

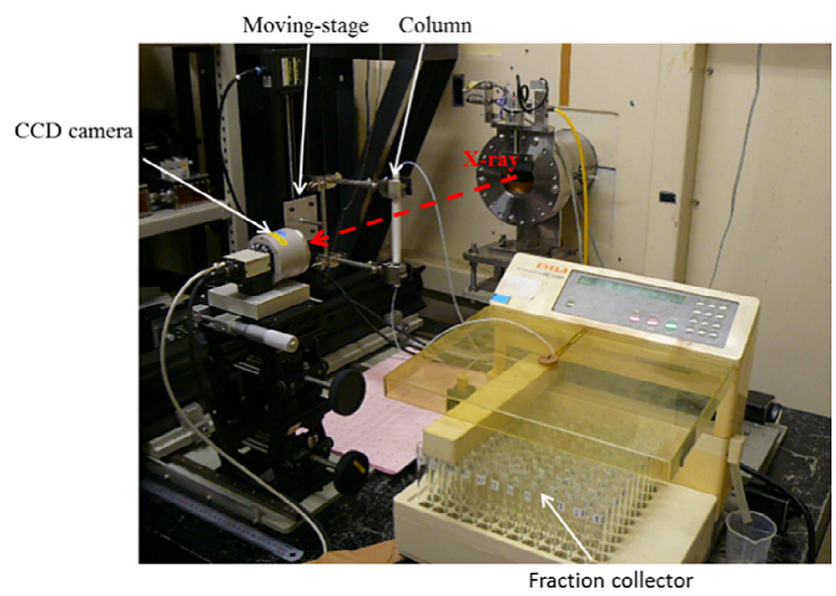

Fig. 5. Experimental setup for the X-ray imaging.

as the extractant by impregnating it into the $\mathrm{SiO}_{2}-\mathrm{P}$ support. The packed columns of $3 \mathrm{~mm} \phi-100 \mathrm{mmH}$ (cylindrical bed) or $3 \mathrm{~mm} \times 10 \mathrm{~mm} \times 100 \mathrm{mmH}$ (rectangular parallelepiped bed) containing the adsorbent were irradiated by $\gamma$-ray at ${ }^{60} \mathrm{Co}$ irradiation facility in Takasaki Laboratory of Japan Atomic Energy Agency. During the irradiation, mobile phase inside the column was continuously supplied with $0.9 \mathrm{~mL} / \mathrm{min}$ or was stopped by closing the line. The irradiation dose rate was $3 \mathrm{kGy} / \mathrm{h}$, and integrated irradiation dose was about 0.1 MGy.

Bubbles produced inside the bed by the irradiation were observed by X-ray imaging. The experiment was carried out at BL27B beamline of Photon Factory in High Accelerator Research Organization, Japan. Experimental setup for the imaging is shown in Figure 5. The incident $\mathrm{X}$-ray obtained from synchrotron radiation was monochromaterized by $\mathrm{Si}(311)$ double crystal to $18.1 \mathrm{keV}$ and then guided inside of the experimental hatch. Intensity of the X-ray passing through the column was measured by the CCD camera. The resolution of the X-ray imaging was about $25 \mu \mathrm{m}$. The column was set at moving-stage, and $\mathrm{X}$-ray image of whole of the column was obtained by 2 min scanning. A pump for supplying solution and a fraction collector for sampling effluent were set at upstream and downstream of the column, respectively. Pump and tanks for the solutions were located outside the experimental hatch.

In order to evaluate influences of the bubbles on the separation performance, column separation experiments using the rectangle columns before and after the irradiation were also carried out. A feed solution $\left(3 \mathrm{M} \mathrm{HNO}_{3}\right.$ containing $\mathrm{Y}(\mathrm{III})$, $\mathrm{Sr}(\mathrm{II})$ and $\mathrm{Zr}(\mathrm{IV}))$, wash solution $\left(3 \mathrm{M} \mathrm{HNO}_{3}\right)$, eluents $\left(\mathrm{H}_{2} \mathrm{O}\right.$ and $50 \mathrm{mM}$ Diethylene Triamine Pentaacetic Acid [DTPA] solution at $\mathrm{pH}=3$ ) were sequentially supplied to the columns, and then effluents were fractionally collected at every $1.2 \mathrm{BV}$ of the column. Concentrations of the cations in the effluents were analyzed by ICP-AES measurements. During the separation experiments on the $\gamma$-ray irradiated column, distributions of $\mathrm{Y}(\mathrm{III})$ and $\mathrm{Zr}(\mathrm{IV})$ inside the column were evaluated from the $\mathrm{X}$-ray absorption intensities in the same way to that described in reference [12]. 


\section{Results and discussion}

\subsection{Behavior of gas in the engineering scale column}

Figure 6 shows amount of the discharged $\mathrm{N}_{2}$ gas plotted as time after the injection of the gas, where the broken line shows total amount of the supplied gas. Although almost all the supplied gas was accumulated inside the column when the flow direction is upward, the downward flow succeeded in discharging large part of the supplied gas. Since the gas inlet is located at the bottom of the column, the distance from the location of the gas to the outlet rather than the direction of the flow even when it is opposite to gravity must be essential for the difference in the results. If gases generate at close to the outlet of the column, almost all of them would be discharged through normal operation.

Although upward flow could not discharge the supplied gas, the accumulated gas was discharged when the upward flow was restarted after stopping the flow. The stop and restart of the upward flow was considered to change the distribution of gas, and then the gas accumulating inside the bed must be discharged. Therefore, switching of the feed pump is expected to be one of the effective methods to discharge the accumulated gas.

The flow velocity distribution inside the column and height equivalent of the theoretical plate (HETP) at the conditions of (a) and (c) were shown in Figure 7, where the flow velocities and the HETPs for the columns without supplying gas were also shown. The HETP at the condition of (c) shows greater value than that evaluated for the column without supplying the gas, whereas HETP of (a) showed little difference from that of without supplying gas. Therefore, accumulated gas may disturb the flow inside the bed. There is distinct difference in the flow velocity between at the center of the column and at close to the wall. The accumulation of gas must be impediment for obtaining the uniform flow. The gases generated by radiolysis have to be discharged with respect to not only the safety but also the separation performance of the column.

\subsection{CFD simulations}

The amount of the gas and increased temperature due to radioactive nuclides were calculated under the condition of $T=323 \mathrm{~K}$ for the initial and ambient temperature and $v=4 \mathrm{~cm} / \mathrm{min}$ for the mobile phase. The generated products were properly dissolved into the mobile phase, and the gases did not accumulate. Temperature inside the column was almost constant, and the heat from adsorption band was transported to the downstream by the flow. Generation rate of the hydrogen from the adsorption band is $3.2 \times 10^{-5} \mathrm{~mol} / \mathrm{dm}^{3} \cdot \mathrm{s}$ and the
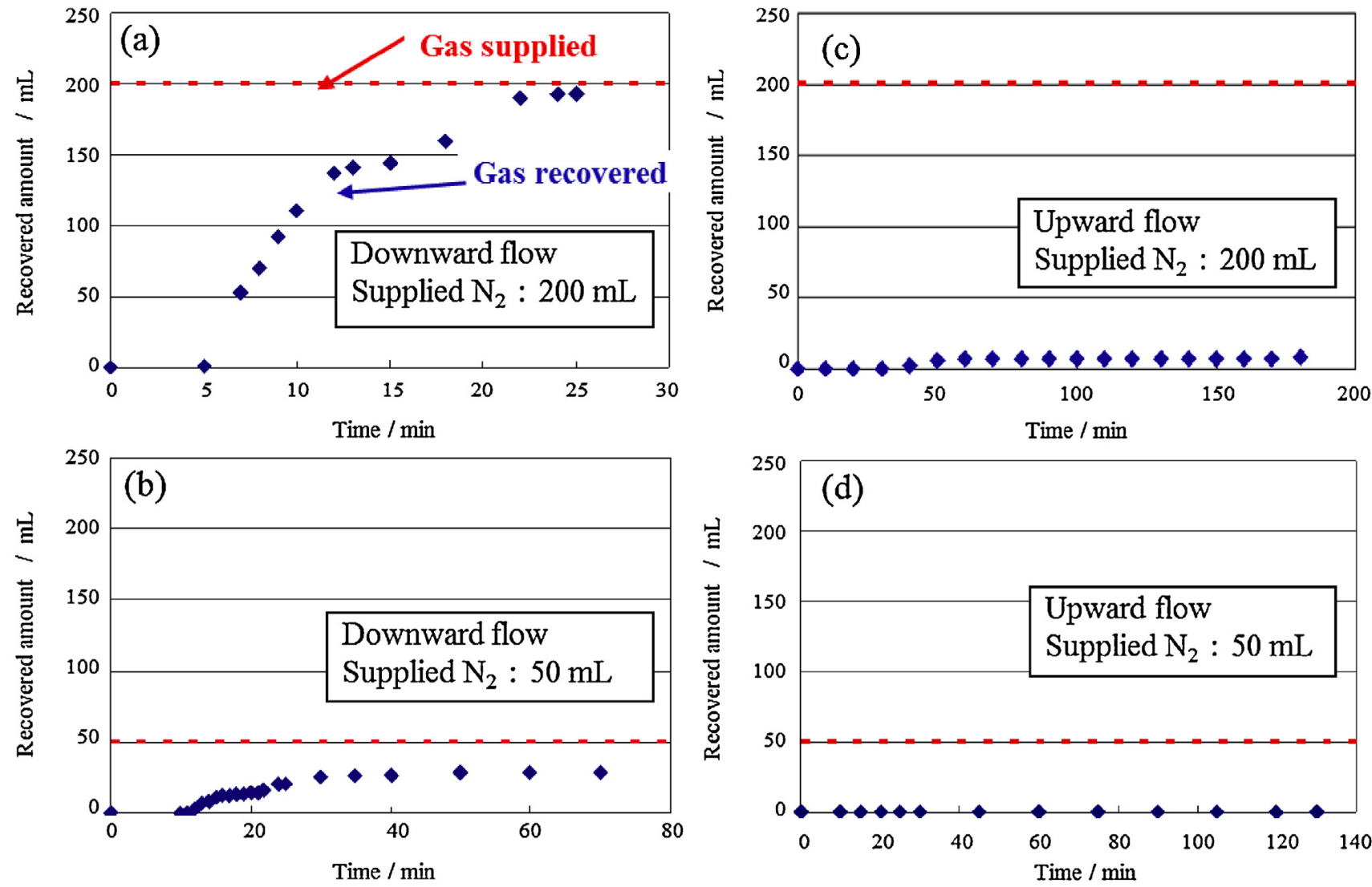

Fig. 6. Amount of recovered gas from the column. (a) Flow direction was downward and amount of supplied $\mathrm{N}_{2}$ was $200 \mathrm{~mL}$; (b) Flow direction was downward and amount of supplied $\mathrm{N}_{2}$ was $50 \mathrm{~mL}$; (c) Flow direction was upward and amount of supplied $\mathrm{N}_{2}$ was $200 \mathrm{~mL}$; (d) Flow direction was upward and supplied $\mathrm{N}_{2}$ was $50 \mathrm{~mL}$. Flow velocity was controlled at $4 \mathrm{~cm} / \mathrm{min}$. 


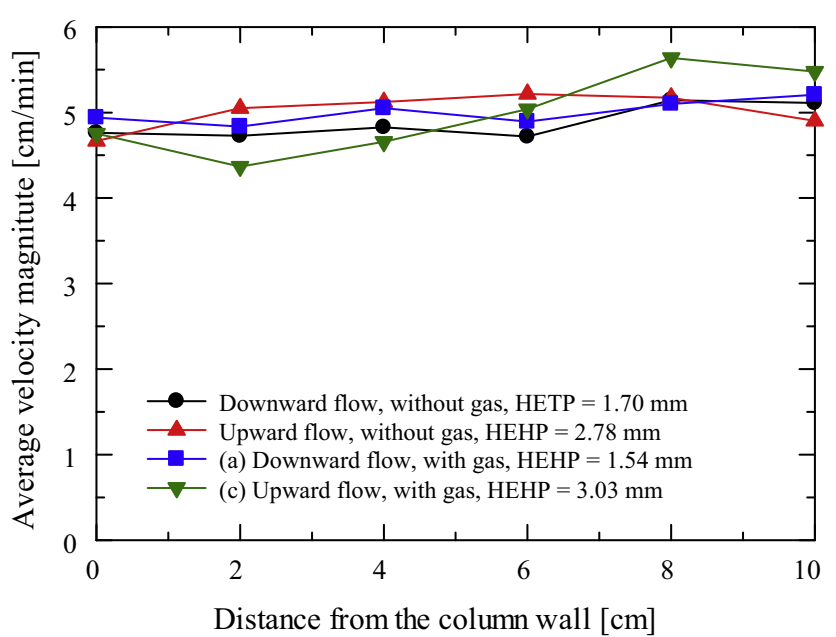

Fig. 7. The flow velocity distribution inside the column $(\mathrm{ID}=20 \mathrm{~cm})$ and HETP.

solubility of the hydrogen gas into the water at 1 atm and $300 \mathrm{~K}$ is ca. $7 \times 10^{-4} \mathrm{~mol} / \mathrm{dm}^{3}$, then the generated products are considered to dissolve into the mobile phase immediately. In the case of $\mathrm{O}_{2}, \mathrm{CO}_{2}$ and $\mathrm{NO}_{2}$, ratios of $\mathrm{G}$ values to the solubility of them into water are smaller than that of hydrogen, so they should dissolve in water as well. Therefore, hydrogen and oxygen do not accumulate inside the column but dissolve into the mobile phase and are discharged with an eluent during the operation.

Figure 8 shows the distribution of the accumulated gas and temperature inside the column at $t=600,3,600 \mathrm{~s}$, where the flow was stopped at $t=0 \mathrm{~s}$. The gas began to accumulate before $t=600 \mathrm{~s}$, and amount of the accumulated gas increased with proceed with time. About $1,700 \mathrm{~mL}(0.15 \mathrm{~mL} / 1 \mathrm{~mL}$ bed $)$ of gases at the standard condition was accumulated at $t=3,600 \mathrm{~s}$. Composition of the gas is $93 \%$ of $\mathrm{H}_{2}$ and $7 \%$ of $\mathrm{O}_{2}$. Generated $\mathrm{CO}_{2}$ and $\mathrm{NO}_{2}$ were properly dissolved into the water. Since the mixture of hydrogen and oxygen shows explosive nature, the accumulated gases should be discharged from the column. The decay heat also accumulated at the adsorption band after the stop of the flow, and wall cooling was effective only at close to the wall. Thermal conductivity of the adsorbents must be too small to remove the decay heat inside the bed only by the wall cooling.

In order to evaluate the performance of chilled eluent for discharging the accumulated gas, the amount of the accumulated gas inside the column after the restart of the flow was calculated. This calculation was started from the state of 3,600 s after the stop of the operation as shown in Figure 8. The flow velocity and temperature of the coolant were $v=16 \mathrm{~cm} / \mathrm{min}$ and $T=278 \mathrm{~K}$, respectively. In this simulation, flow velocity and temperature of the mobile phase were changed from those for the normal operation in order to enhance the dissolution of the gas into the mobile phase. The accumulated gas and gas generated from the adsorption band were gradually dissolved into the coolant, and they were entirely discharged from the column at $t=1,020 \mathrm{~s}$. The accumulated heat was simultaneously discharged from the column by the coolant. Since a part of gas accumulating at the lower part of the column could

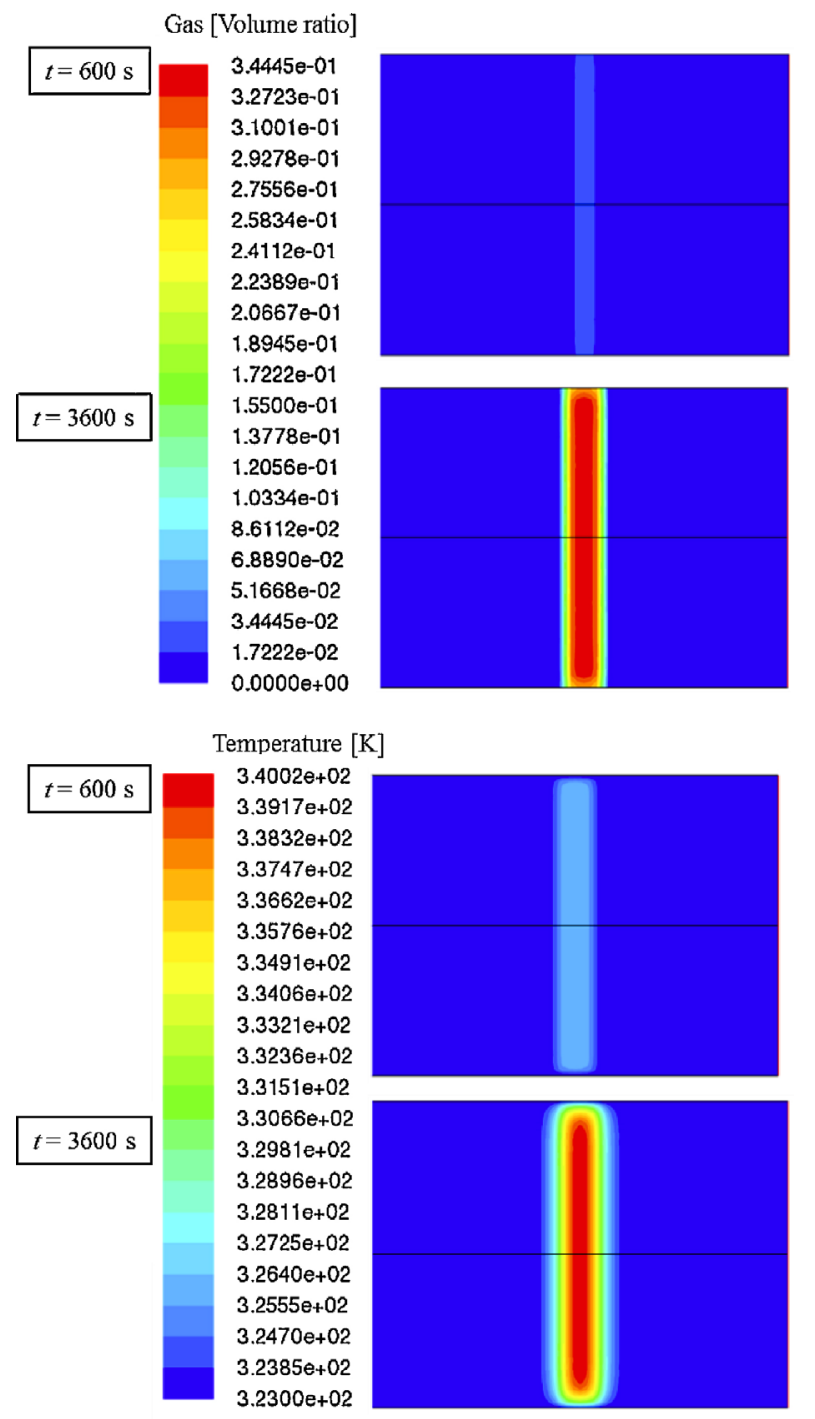

Fig. 8. Volume ratio of gas and temperature inside the column.

be pushed out by the mobile phase as seen in the previous section, it must be required shorter time to discharge the gas accumulated. An equipment for supplying the emergency coolant which consists of pumps, tanks and pipes is important for the safety of the system.

\subsection{X-ray imaging on $\gamma$-ray irradiated columns}

Figure 9 shows X-ray image of the cylindrical columns. Bubbles generated by the external irradiation inside the bed were not confirmed in the images of the unirradiated column and of the irradiated column with the flow of the mobile phase. This result agrees with those obtained by the CFD simulation described in the previous section, and radiolytically generated hydrogen and oxygen should be dissolved in the mobile phase and be discharged. On the other hand, small bubbles with the size of $\sim 0.3 \mathrm{~mm}$ ununiformly distributed inside the bed of the column irradiated without the flow of the mobile phase. As well as in the cylindrical column, bubbles were observed in the 


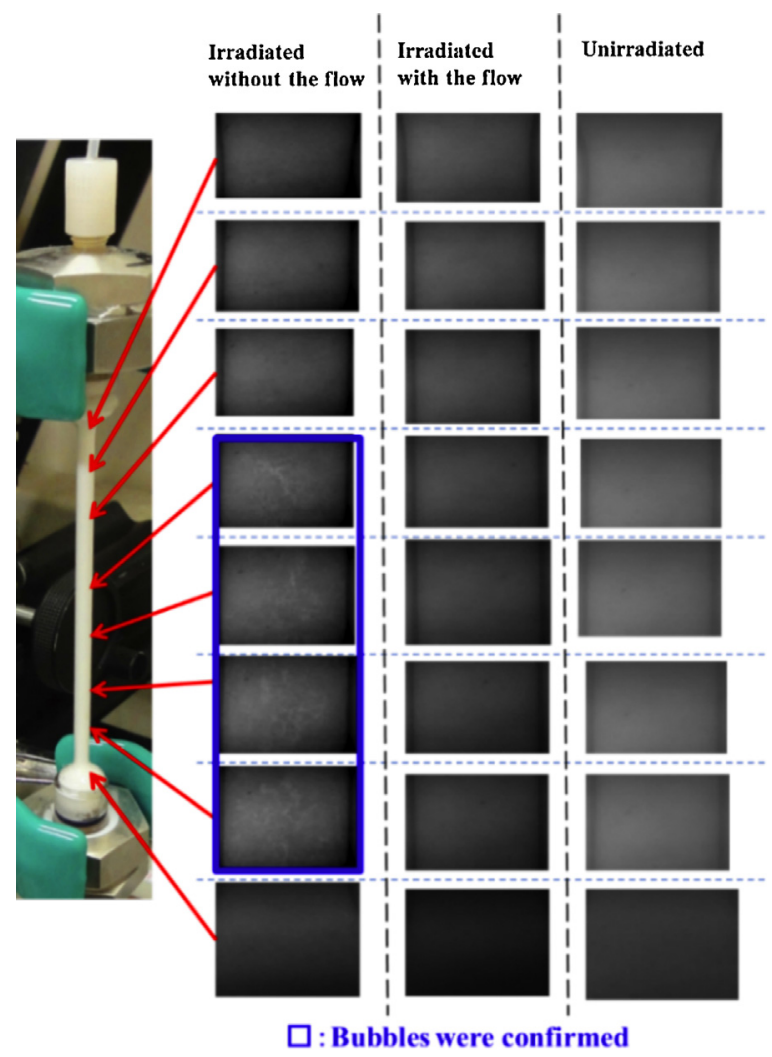

Fig. 9. X-ray image of the $\gamma$-ray irradiated cylindrical columns.

irradiated rectangle column without the flow. Water was supplied to the irradiated rectangle column with $0.9 \mathrm{~mL} /$ $\min (v=3 \mathrm{~cm} / \mathrm{min})$ to observe the behavior of the accumulated gas. Figure 10 shows bubbles in the rectangle column after start of the flow. The number of the bubbles decreased after supplying the mobile phase into the bed. This result must correspond to the dissolution of the accumulated gas into the coolant observed in the CFD simulation. Although the CFD employed a quite simple model and carried out a conservative estimation, accumulation and dissolution behavior of the gas must be qualitatively reproduced in the simulation. In addition to that, bubbles moving with the effluent were also observed at the downstream of the column. This result supports the experimental results obtained for the large scale column system. Therefore, supplying the mobile phase is effective not only to dissolving the gas inside the column but also to pushing the gas away from the column. However, the bubbles at near the wall of the column stayed even after the start of the flow. Decrease in the flow velocity near the corner of the rectangle column is suspected to lead the remaining bubbles.

Elution curves obtained for the unirradiated rectangle column packed with $\mathrm{CMPO} / \mathrm{SiO}_{2}-\mathrm{P}$ adsorbent are shown in Figure 11. As shown in the previous report [3], $\mathrm{Sr}$ (II) was not extracted by CMPO and was eluted in the effluents. Y(III) is adsorbed and eluted by supplying $\mathrm{H}_{2} \mathrm{O}$ and the wash solution $\left(3 \mathrm{M} \mathrm{HNO}_{3}\right)$ [13]. $\mathrm{Zr}$ (IV) adsorbed by CMPO was retained and then eluted with the DTPA solution. Those not general elution behaviors are considered to be caused by mixing solutions in the relatively long flow channel between the

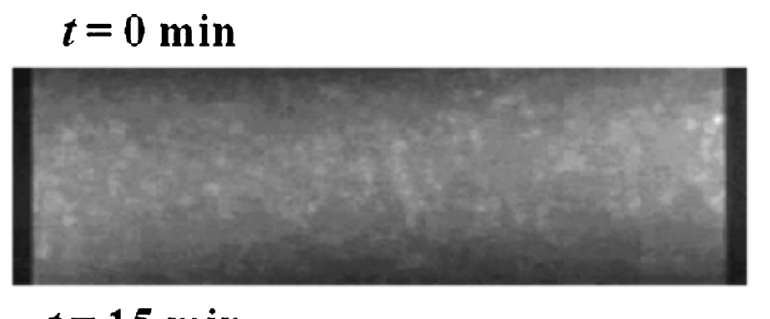

\section{$t=15 \mathrm{~min}$}

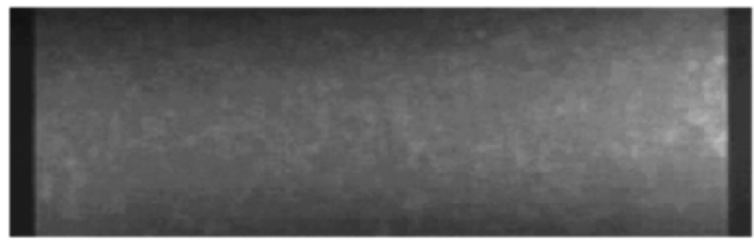

\section{$t=25 \mathrm{~min}$}

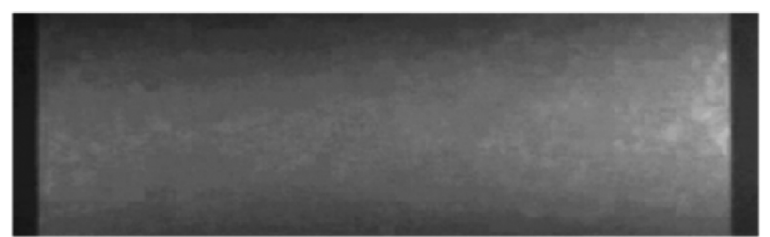

Fig. 10. X-ray image of the $\gamma$-ray irradiated rectangle column after restart of the flow of $3 \mathrm{~cm} / \mathrm{min}$.

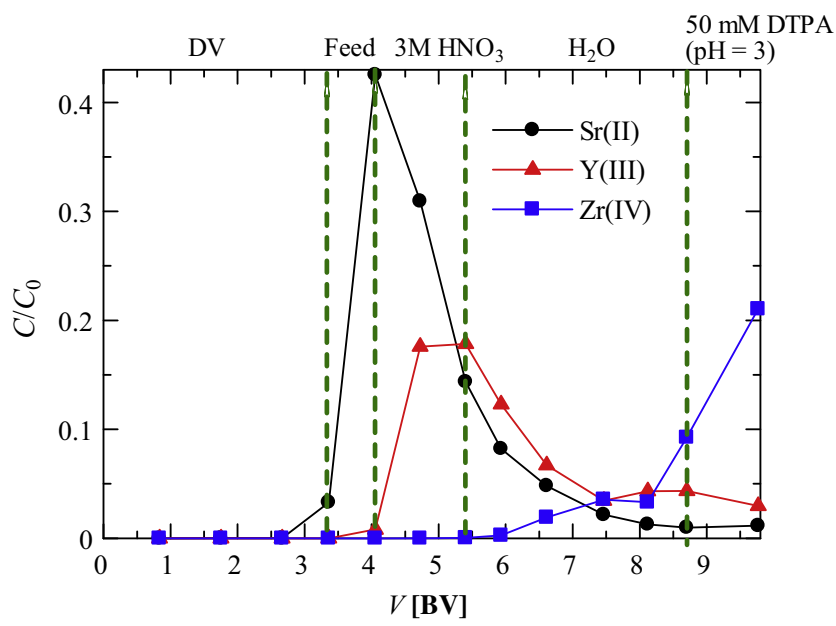

Fig. 11. Chromatogram obtained for the unirradiated rectangle column.

column and tanks located at the outside of the experimental hatch.

Figure 12 shows the elution curves obtained for the $\gamma$-ray irradiated rectangle column with the deposited gas. Elution behavior of Sr did not seem to be affected by the irradiation. Elution of $\mathrm{Y}(\mathrm{III})$ and $\mathrm{Zr}(\mathrm{IV})$ began faster than those observed for the unirradiated column. Degradation of $\mathrm{CMPO} / \mathrm{SiO}_{2}-\mathrm{P}$ adsorbent would not be significant for 0.1 MGy irradiation [14], however, the change was observed as shown in Figure 12 and this was attributed to the influence of degradation of CMPO, where adsorption of Y(III) which shows weak interaction with CMPO was apparently suppressed. 


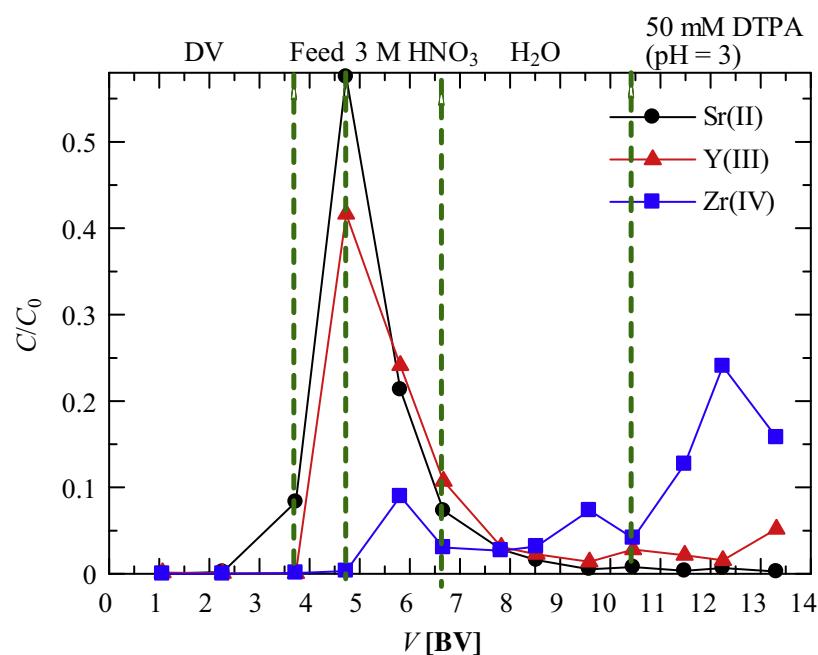

Fig. 12. Chromatogram obtained for the $\gamma$-ray irradiated rectangle column.

Concentrations of $\mathrm{Y}(\mathrm{III})$ and $\mathrm{Zr}(\mathrm{IV})$ during the separation operation using the $\gamma$-ray irradiated rectangular column are shown in Figures 13 and 14, respectively. Color strengths correspond to the concentrations of cations, and $V$ in the figure corresponds to $V$ in Figure 12. For the ideal column operation, rectangle shape adsorption band is expected to move to the downstream of the column as the progress of time. However, distributions of $\mathrm{Y}(\mathrm{III})$ and $\mathrm{Zr}(\mathrm{IV})$ inside the column are not uniform in the laterally direction of the column as suggested from the elution curves. This indicates that flow velocity inside the bed was not uniform. Obstruction of the flow by the bubbles is considered to induce flow paths of the mobile phase inside the bed.

Consequently, the bubbles radiolytically generated disturb the uniform flow inside the bed as seen in Section 3.1. The non-uniform flow inside the bed may lead the non-uniform distribution of acidity or of DTPA concentration inside the bed. Besides degradation of CMPO extractant, those nonuniform distributions of them are considered to result in the

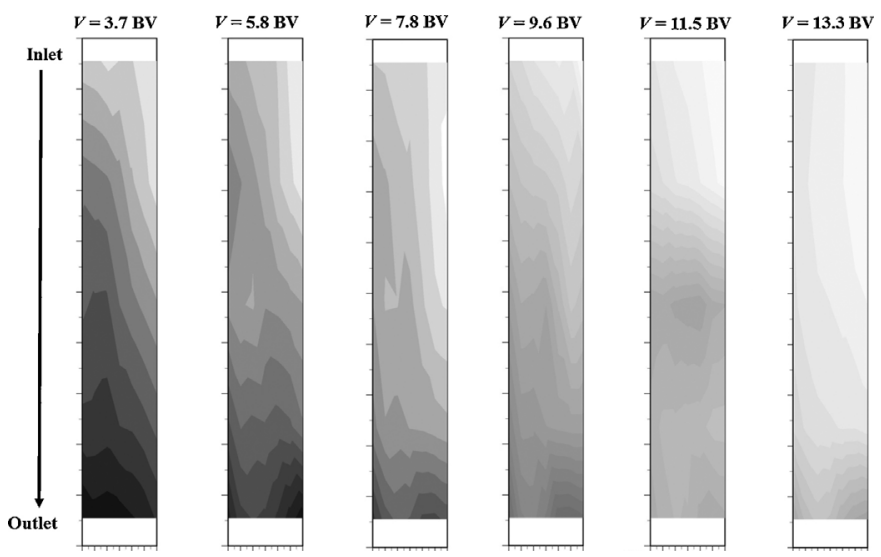

Fig. 13. Concentration profile of Y(III) during the separation operation with the rectangular column irradiated with $\gamma$-ray in advance.
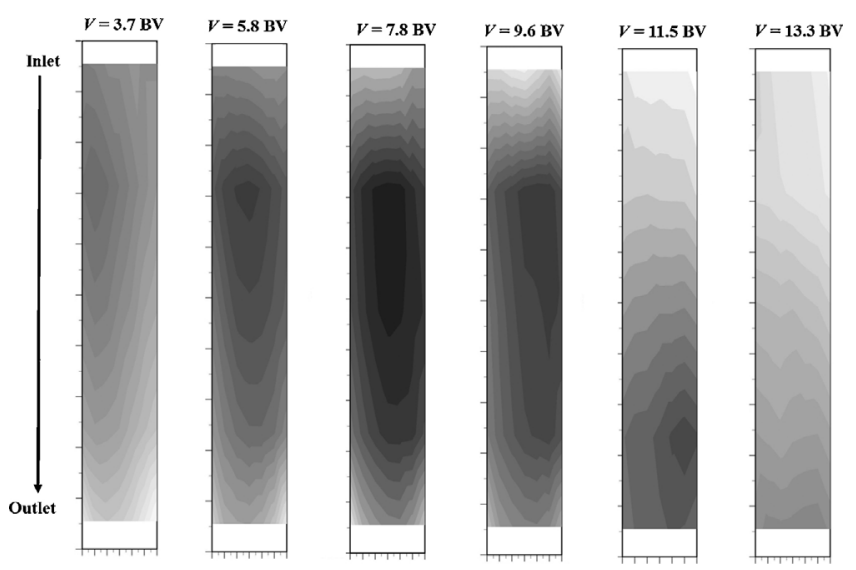

Fig. 14. Concentration profile of $\mathrm{Zr}(\mathrm{IV})$ during the separation operation with the rectangular column irradiated with $\gamma$-ray in advance.

faster elution of $\mathrm{Y}(\mathrm{III})$ and $\mathrm{Zr}(\mathrm{IV})$. Accumulated gas is revealed to influence on the separation performance of the column, thus preliminary operation to discharge the gas and to recover the uniform flow is important even after a short period of unexpected stop of the system.

\section{Conclusions}

Generation, accumulation and discharge behavior of hydrogen gas radiolytically generated inside the extraction chromatography column were investigated through experiments with large scale column system, Computation Fluid Dynamics (CFD) simulation and X-ray imaging experiments on $\gamma$-ray irradiated column. Although both heat and gas accumulate at the adsorption band after the stop of the operation, supply of a coolant was revealed to be effective to discharge them. Bubbles inside the bed obstruct uniform flow inside the bed due to formation of flow paths. The accumulated gas should be discharged not only to secure safety of the system but also to guarantee the column performance. In the practical system, an equipment for feeding a coolant is effective.

This work was financed by the Ministry of Education, Culture, Sports, Science and Technology of Japan (MEXT) under the framework of "The Development of Innovative Nuclear Technologies". X-ray imaging experiments were carried out under the proposals 2010G047 of the Photon Factory, KEK.

\section{Nomenclature}

$v \quad$ flow velocity inside the column

HETP height equivalent of a theoretical plate

$\mathrm{BV} \quad$ volume of the packed bed

$\mathrm{SiO}_{2}-\mathrm{P}$ porous silica support coated by styrene divinyl benzene co-polymer

CMPO n-octyl(phenyl)- $N, N$-diisobutylcarbamoyl-methylphosphine oxide

DTPA diethylenetriaminepentaacetic acid 


\section{References}

1. E.P. Horwitz, M.L. Dietz, R. Chiarizia, H. Diamond, S.L. Mazwell III, M.R. Nelson, Separation and preconcentration of actinides by extraction chromatography using a supported liquid anion exchanger: application to the characterization of high-level nuclear waste solutions, Anal. Chim. Acta 310, 63 (1995)

2. Y. Koma, Y. Sano, K. Nomura, S. Watanabe, T. Matsumura, Y. Morita, Development of the extraction chromatography system for separation of americium and curium, in Proceedings OECD Nuclear Energy Agency 11th Information Exchange Meeting on Actinide and Fission Product Partitioning and Transmutation, San Francisco, USA, November 1-4, 2010, IV-4, OECD/NEA (2010)

3. S. Watanabe, T. Senzaki, A. Shibata, K. Nomura, Y. Koma, Y. Nakajima, MA recovery experiments from genuine HLLW by extraction chromatography, in Proceedings Global 2011, Makuhari, Japan, December 11-16, 2011, paper 387433, Atomic Energy Society of Japan (CD-ROM) (2011)

4. S. Watanabe, I. Goto, Y. Sano, Y. Koma, Chromatography column system with controlled flow and temperature for engineering scale application, J. Eng. Gas Turbines Power 132, 102903 (2010)

5. S. Watanabe, I. Goto, K. Nomura, Y. Sano, Y. Koma, Extraction chromatography experiments on repeated operation using engineering scale column system, Energy Procedia 7, 449 (2011)

6. Y.-Z. Wei, K.N. Sabharwal, M. Kumagai, T. Asakura, G. Uchiyama, S. Fujine, Studies on the separation of minor actinides from high-level wastes by extraction chromatogra- phy using novel silica-based extraction resins, Nucl. Technol. 132, 413 (2000)

7. Research Group for Aqueous Separation Process Chemistry, Nuclear Science and Engineering Directorate, Japan Atomic Energy Agency, JAEA-Review2008-037 Handbook on process and chemistry of nuclear fuel reprocessing. Version 2 (Japan Atomic Energy Agency, 2008), p. 530

8. Y. Koma, S. Watanabe, Y. Sano, T. Asakura, Y. Morita, Extraction chromatography for $\mathrm{Am}$ and $\mathrm{Cm}$ recovery in engineering scale, in Proceedings ATALANTE 2008: nuclear fuel cycles for a sustainable future, Montpellier, France, May 19-23, 2008; O1-19 (CD-ROM) (2008)

9. R. Sander, Compilation of Henry's law constants for inorganic and organic species of potential importance in environmental chemistry. Version 3, http://www.henrys-law.org (1999)

10. ANSYS Inc, GAMBIT 2.4 user's guide, 2007

11. ANSYS Inc, FLUENT 12.0 user's guide, 2009

12. S. Watanabe, Y. Sano, M. Myouchin, Y. Okamoto, H. Shiwaku, A. Ikeda-Ohno et al., In-situ analysis of chemical state and ionic distribution in the extraction chromatography column, J. Ion Exchange 21, 73 (2010)

13. Y. Wei, A. Zhang, M. Kumagai, M. Watanabe, N. Hayashi, Development of the MAREC process for HLLW partitioning using a novel silica-based CMPO extraction resin, J. Nucl. Sci. Technol. 41, 315 (2004)

14. S. Watanabe, S. Miura, Y. Sano, K. Nomura, Y. Koma, Y. Nakajima, Alpha-ray irradiation on adsorbents of extraction chromatography for minor actinides recovery, in Proceedings OECD Nuclear Energy Agency 12th Information Exchange Meeting on Actinide and Fission Product Partitioning and Transmutation, Prague, Czech Republic, 24-27 September 2012, V-31, OECD/NEA (2013)

Cite this article as: Sou Watanabe, Yuichi Sano, Kazunori Nomura, Yoshikazu Koma, Yoshihiro Okamoto, Safety operation of chromatography column system with discharging hydrogen radiolytically generated, EPJ Nuclear Sci. Technol. 1, 9 (2015) 